\title{
On the role of blank spaces for eye-movement control in visual search
}

\author{
ARTHUR M. JACOBS \\ Groupe Regard, Laboratoire de Psychologie Experimentale, Paris, France
}

\begin{abstract}
The present experimental data demonstrate that in a simple letter-search task using a highly repetitive spatial pattern, the use of a block structure similar to the one we are familiar with in a reading situation (blocks of characters separated by blank spaces) does not facilitate scanning behavior. The presence of blank spaces interspersed irregularly within lines of $x \mathrm{~s}$ which have to be scanned in search of a target letter causes a decrease in fixation durations but also decreases saccade size. The results can be understood in terms of an inhibition of visual processing by the presence of the blank spaces: the visual span for the different target letters was found to be significantly smaller when blank spaces were present within the background than when they were absent. Saccade sizes adapt to this change in visual span and become smaller, confirm. ing the visual-span-control hypothesis for scanning eye movements (Jacobs, 1986). When saccades become smaller, less information is extracted at each fixation, causing a decrease in processing times and shorter fixation durations.
\end{abstract}

In visual search and reading, the eyes move rapidly from one fixation point to the next and the brain has to compute efficiently at each fixation the next landing position of the eye ("where" decision) as well as the moment when the saccade occurs ("when" decision). These "where" and "when" decisions of eye-movement control can be influenced directly by a variety of perceptual and cognitive factors, depending on the difficulty of the task (see McConkie, 1983, O'Regan \& Lévy-Schoen, 1987, and Rayner, 1984, for recent reviews on this issue). One perceptual factor directly involved in determining the computation of the next landing position of the eye during reading are the blank spaces between words (Pollatsek \& Rayner, 1982). Other perceptual factors known to have an effect on saccade control in reading, such as word length (O'Regan, 1979), can have this effect only because blank spaces perceptually isolate the processing unit which presumably is the most important for eye-movement control in reading: the word (McConkie \& Zola, 1984). If the blank spaces between words are masked out (Brady, 1981; Fisher, 1975; Menz \& Groner, 1985) or if their visibility in the parafovea is decreased ( $\mathrm{O}^{\prime}$ Regan, LévySchoen, \& Jacobs, 1983), reading rate slows down significantly. Although the importance of blank spaces for eye-movement control is clear with regard to reading, their role in eye guidance in visual search seems much less well understood (Jacobs \& O'Regan, in press; see also Moffitt, 1980, for a review). The present study therefore investigates if and how blank spaces influence eye

This research was supported by a grant from the FYSSEN Foundation, Paris. I would like to thank all members of the Groupe Regard, especially Ariane Lévy-Schoen and J. K. O'Regan, for their helpful discussions. I am also grateful to two anonymous reviewers and to Professor Eriksen for their useful comments. Requests for reprints should be sent to: Arthur M. Jacobs, Groupe Regard, Laboratoire de Psychologie Expérimentale, 28 rue Serpente, 75006 Paris, France. movement control during visual search. Using a lettersearch task like the one used by Jacobs (1986), in which information load is reduced to the very minimum, the primary question is whether or not the use of a global spatial structure similar to the one we are familiar with in reading-namely the use of blocks of characters of variable size separated by blank spaces-will facilitate search behavior.

For a letter-search task in which the global spatial structure was continuous, namely, uniform lines of $x \mathrm{~s}$ with the occasional insertion of the sought-for target letter, it has been shown recently that saccade size depends on the variable size of the visual span of the sought-for target (Jacobs, 1986). The data indicated that, on average, the eyes jump, at each fixation during search, to the limit of the visual span of the given target letter. (Visual span was defined as the maximum eccentricity from the eye's fixation point at which a given target letter within a background pattern can be detected with a certain probability.) Thus, one can assume that the processing unit functional in scanning a repetitive background pattern in search of a known target is the visual span of the target. If blank spaces were inserted irregularly within such a repetitive pattern, thereby forming separate perceptual units (blocks of characters) within the visual span, the following question would arise: Will the system still function in a visual-spancontrol mode in which saccade size adjusts directly to the visual span of the sought-for target, or does this mode break down because of the adoption of a global scanning strategy of the type "at each fixation jump to the next fairly long character block visible in parafovea'"?

Let us consider the examples of Figure 1, which gives an illustration of the material used in the following experiments. A visual-span-control mode would predict strong changes in saccade size for the two target letters, which differ in their graphic similarity with the back- 


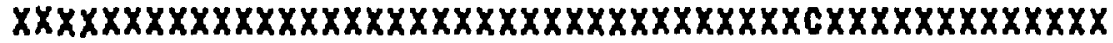

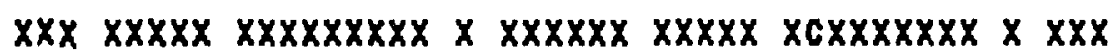
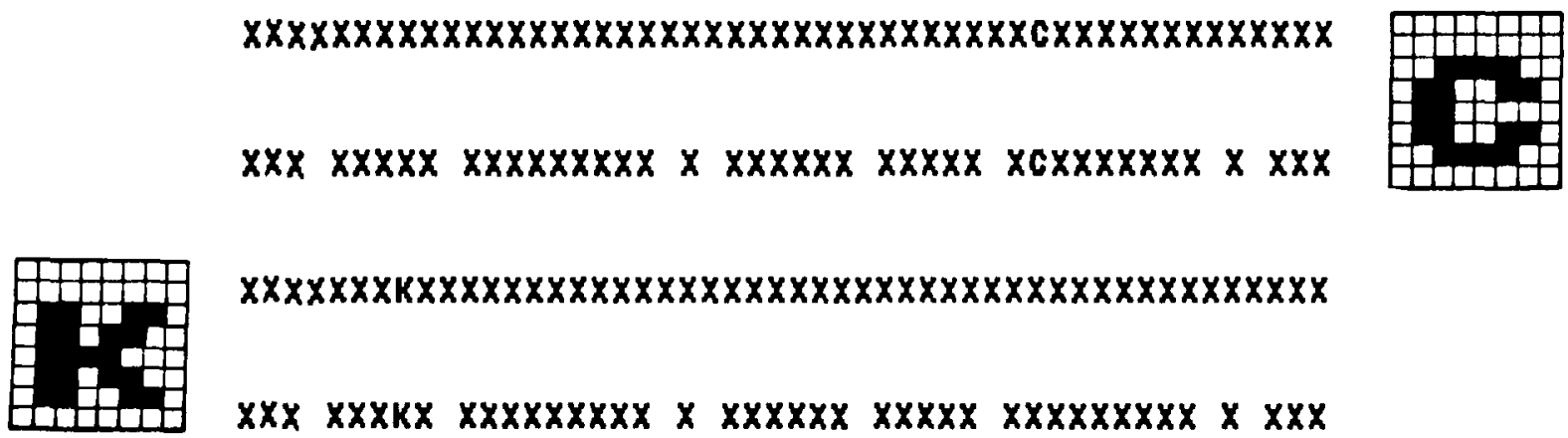

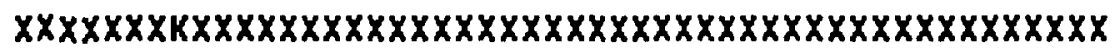

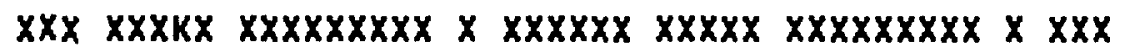

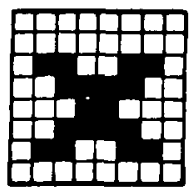

Figure 1. Example of stimulus lines used in the letter-search task. Lines 1 and 3 are examples of the no-space condition; lines 2 and 4 are examples of the space condition. The upper two lines contain the dissimilar target letter $c$, which is easily detectable in peripheral vision (large visual span); the lower lines contain the similar target letter $k$ which has a small visual span. For purposes of clarity, the letter matrices of the two target letters are also given in comparison with the matrix of the background $x$. Note that the stimulus lines used were actually longer than indicated in the above examples ( 70 characters).

ground $x \mathrm{~s}$ and in the size of their visual span (Jacobs, 1986). This should be true for both the normal (lines 1 and 3 ) and the space condition (lines 2 and 4 ). In the space condition, a pure-block-control mode, however, should yield no important differences between the saccade sizes for the $c$ and the $k$ targets, if the mean number of blocks per line is kept constant for both conditions.

Together with this question about saccade control, a second, interesting issue investigated here concerns the control of fixation duration. In Jacobs (1986), fixation durations were relatively long (mean fixation duration increased up to $520 \mathrm{msec}$ ). This is about two times as much as the normal fixation duration found in reading, and points to the need to identify which mechanism(s) take so much time in a task in which cognitive load was reduced to the very minimum. Now, one reason that might explain part of these considerably longer fixation durations could be that in this situation the uniform spatial pattern made it very difficult for the brain to know which region of the line had already been attended to and which had not, thus delaying saccade programming. The idea tested here is simple: If a global structure is added to the repetitive spatial pattern by interspersing blank spaces into the lines of $x \mathrm{~s}$, thereby providing feedback about relative gaze positions within the line, this might facilitate saccade programming and result in shorter fixation durations for the space condition.

\section{EXPERIMENT 1: SCANNING BEHAVIOR STUDY}

\section{Method}

Subjects. Eight students of experimental psychology at the University René Descartes in Paris participated in this experiment. All had normal vision and were naive as to the purpose of the study.
Stimuli. As illustrated in Figure 1, a set of lines of $x \mathrm{~s}$ was used within which a known target letter was hidden at randomly varying positions. Note that in reality the lines of $x$ s were 70 characters long and not as short as illustrated in Figure 1. Each set was made up of 15 lines; the first line always contained the sought-for target letter, and only 4 other lines, chosen at random, contained one or two target letters. The other 10 lines contained only $x \mathrm{~s}$, and only these lines were selected for data analysis. Please note that only one type of target letter was presented within the same set of lines, and that the subjects were informed of what the target was. Thus, any effects due to confusion between the two possible targets were prevented; we were interested in the influence of global spatial structure on eye movements, and not in the effects of positive or negative set size. The two levels of target-background similarity (target letters) were chosen for purposes of comparison with Jacobs (1986).

In the space condition (see Figure 1, lines 2 and 4), the lines of $x \mathrm{~s}$ were broken up into blocks of varying length $(1,3,5,7,9$, or 11 characters long). Target letters were inserted at random positions within the given lines, with the constraints that a target never appear at the beginning or the end of a block and that a target not constitute a single-letter block. Odd numbers of characters were used in the blocks so that the targets were always flanked by at least one $x$. On average, there were 13 blocks to a line, with their lengths chosen at random by the computer. Mean block length was 5 characters, the shorter block lengths $(1,3$, and 5 characters) were used twice as frequently as the longer ones, thus somewhat approximating the spatial structure of texts in reading. Mean number per line and length of blocks were kept constant for both target conditions.

Apparatus and Experimental conditions. The experiment was controlled by a BBC and an IMS microcomputer. Stimuli appeared on a VELEC VS display terminal using a fast P4 phosphor. The character matrices of the BBC computer were of $8 \times 8$ pixels; their width subtended $1 / 3^{\circ}$ of visual angle at a viewing distance of $60 \mathrm{~cm}$, which was chosen for comparison with the data of Jacobs (1986). Lighting conditions were photopic and were kept identical across subjects, as were screen brightness and contrast. The display background luminance was about $0.7 \mathrm{~cd} / \mathrm{m}^{2}$; stimulus luminance was about $70 \mathrm{~cd} / \mathrm{m}^{2}$.

The subject's eye movements were monitored by a photoelectric scleral reflection technique and analyzed by the computer, which 
sampled eye position at $100 \mathrm{~Hz}$. Automatic identification of saccades and fixations was done according to the following principle: a saccade was defined as a change in eye position of more than one character space that took less than $50 \mathrm{msec}$ and gave rise to a fixation lasting more than $50 \mathrm{msec}$. While the subject was scanning the lines, the computer continuously corrected an initially done calibration for slow shifts caused by head movements or other artifacts (see O'Regan et al., 1983, or Jacobs, 1986, for more details on the eye-movement analysis program). Relative accuracy of estimation of the size of the saccades was guaranteed to be the same across subjects and conditions and was of the order of one character space. The display technique used was the same as that used by Jacobs (1986), in which the subject's return movement triggers the disappearance of the line just scanned and the appearance of the next line.

Procedure. After an initial calibration phase, the subject, sitting in an adjustable chair with his or her head stabilized by a chin/ foreheadrest, scanned the set that appeared, line by line, on the computer screen. The subject was asked to scan the lines from left to right, just as in reading, and to count the number of target letters that occurred without missing any. After each experimental block, the correctness of the subject's performance, in terms of number of targets detected, was assessed. To discourage inattentive scanning behavior, the subjects were told that the experimental run would have to be repeated if there were omissions. Thus, the instructions stressed accuracy of performance, rather than speed.

Experimental design. The experimental design was a typical twofactors repeated measures design (subjects $\times$ target $\times$ spatial struc- ture). Two targets, the dissimilar $c$ and the similar $k$, were used, as well as two global spatial structures (block and no-block conditions; see Figure 1). The two targets were the same as those used by Jacobs (1986). The order of presentation followed a balanced Latin square design, in which each subject served in all four conditions ( 2 targets $\times 2$ spatial structures) but in different orders.

\section{Results and Discussion}

Of interest here was ocular behavior while the lines were being scanned in search of an expected target, and not the adjustments of the eyes when a target fell within the peripheral field of view. Therefore, only the lines containing no target letters were selected for analysis, as in Jacobs (1986). On average, the subjects made 0.7 omissions per experimental run (there were, on average, eight targets per run), independently of the condition. The different eye-movement parameters summarized in Table 1 were computed for each subject and each experimental condition, and a two-factor, repeated measures analysis of variance was done. The values for mean saccade size are given in numbers of character spaces, including the blank spaces in the space condition. The results are given in Table 1.

Analysis of spatial eye movement control. The first question posed in this paper concerned the visual span con-

Table 1

la: Results for Different Eye-Movement Parameters as a Function of Target-Background Similarity and Spatial Structure (8 Subjects)

\begin{tabular}{|c|c|c|c|c|c|c|c|c|}
\hline & \multicolumn{8}{|c|}{ Experimental Condition } \\
\hline & \multicolumn{2}{|c|}{$\begin{array}{c}\text { Target } c \\
\text { No Spaces } \\
\end{array}$} & \multicolumn{2}{|c|}{$\begin{array}{c}\text { Target } c \\
\text { With Spaces }\end{array}$} & \multicolumn{2}{|c|}{$\begin{array}{r}\text { Target } k \\
\text { No Spaces } \\
\end{array}$} & \multicolumn{2}{|c|}{$\begin{array}{c}\text { Target } k \\
\text { With Spaces }\end{array}$} \\
\hline & Mean & $S E$ & Mean & $S E$ & Mean & $S E$ & Mean & $S E$ \\
\hline Fixations per Line & 6.8 & & 9.2 & & 13.5 & & 14.2 & \\
\hline Progressions per Line & 5.4 & & 7.4 & & 10.9 & & 11.5 & \\
\hline Regressions per Line & 1.4 & & 1.9 & & 2.5 & & 2.6 & \\
\hline $\begin{array}{l}\text { Progression Size } \\
\text { (in character spaces) }\end{array}$ & 16.5 & 1.3 & 11.2 & 1.2 & 9.0 & .8 & 7.0 & .5 \\
\hline $\begin{array}{l}\text { Fixation Duration } \\
\text { (in milliseconds) }\end{array}$ & 350 & 21 & 318 & 14 & 418 & 11 & 363 & 11 \\
\hline $\begin{array}{l}\text { Search Rate } \\
\text { (items per second) }\end{array}$ & 29.5 & & 24.5 & & 13.7 & & 14.5 & \\
\hline $\begin{array}{l}\text { Total Search Time } \\
\text { (in seconds) }\end{array}$ & 28.1 & & 32.5 & & 61.1 & & 57.2 & \\
\hline \multicolumn{9}{|c|}{ 1b: Results of the ANOVA } \\
\hline & \multicolumn{4}{|c|}{ Factor: Target } & \multicolumn{4}{|c|}{ Factor: Spaces } \\
\hline Fixations per Line & \multicolumn{4}{|c|}{$F(1,7)=95.6, p<.001$} & \multicolumn{4}{|c|}{$F(1,7)=2.3$, n.s. } \\
\hline Progressions per Line & \multicolumn{4}{|c|}{$F(1,7)=135, p<.001$} & \multicolumn{4}{|c|}{$F(1,7)=2.5$, n.s. } \\
\hline Regressions per Line & \multicolumn{4}{|c|}{$F(1,7)=12.4, p<.01$} & \multicolumn{4}{|c|}{$F(1,7)=3.5$, n.s. } \\
\hline $\begin{array}{l}\text { Progression Size } \\
\text { (in character spaces) }\end{array}$ & \multicolumn{4}{|c|}{$F(1,7)=50, p<.001$} & \multicolumn{4}{|c|}{$F(1,7)=11.4, p<.025$} \\
\hline $\begin{array}{l}\text { Fixation Duration } \\
\text { (in milliseconds) }\end{array}$ & \multicolumn{4}{|c|}{$F(1,7)=11.7, p<.025$} & \multicolumn{4}{|c|}{$F(1,7)=12.6, p<.01$} \\
\hline $\begin{array}{l}\text { Search Rate } \\
\text { (items per second) }\end{array}$ & \multicolumn{4}{|c|}{$F(1,7)=26.6, p<.005$} & \multicolumn{4}{|c|}{$F(1,7)=.56$, n.s. } \\
\hline $\begin{array}{l}\text { Total Search Time } \\
\text { (in seconds) }\end{array}$ & \multicolumn{4}{|c|}{$F(1,7)=94.7, p<.001$} & \multicolumn{4}{|c|}{$F(1,7)=.002$, n.s. } \\
\hline
\end{tabular}

Note-All interactions were not significant, except for progression size $(p<.001)$ and search rate $(p<.001)$ 
trol hypothesis (Jacobs, 1986; O'Regan et al., 1983). It asked whether the use of the block structure in the space condition would modulate the visual-span-control strategy that had been observed in a similar scanning experiment (Jacobs, 1986). The data summarized in Table 1 indicate that, as in the latter study, a visual-span-control strategy was adopted by the subjects in the no-space condition, with mean saccade size decreasing clearly as targetbackground similarity increased $(p<.001)$, thus forcing visual span to decline. Second, in the space condition, too, there was an effect of target-background similarity on mean saccade size, indicating the use of a visual span control strategy. However, in the space condition, the eye tended to make more fixations and mean saccade size decreased significantly relative to performance in the no-space condition $(p<.025)$. This suggests that rather than a pure visual-span-control strategy, a mixed strategy that combined visual span control and block control was adopted here. However, it might also be true that, for some reason, visual span was smaller in the space condition than in the no-space condition, thus explaining the observed decrease in saccade size effected by a pure visual span control. To determine which of the two possible interpretations was right, an experiment that quantified visual span for the space and the no-space conditions was needed (see Experiment 2).

Analysis of temporal eye movement control. The result showing that mean fixation duration increased with target-background similarity $(p<.025)$ is entirely compatible with Jacobs's (1986) data and also with the findings of Rayner and Fisher (1987) and Nattkemper and Prinz (1987), who used a similar letter-search task. This indicates that knowledge of the sought-for target letter and its visual properties directly influences the time spent at each fixation to elaborate the "where" and "when" decisions of eye-movement control. Note here that the eyemovement data came from physically identical lines of $x \mathrm{~s}$, since data only from lines that contained no target at all were selected for analysis. Hence, retinal information was the same in the $c$ and the $k$ target conditions, and decisional rather than sensory processes were modulated by the factor "target-background similarity" (see general discussion below; Jacobs, 1987). Table 1 also shows that mean fixation duration decreased by about 30 to $50 \mathrm{msec}$ in the space condition $(p<.01)$, independently of the target letter. In an interpretation of this result, two main lines of reasoning seem possible: The first is given in paragraph 1 below in terms of a facilitation of saccade programming; the second is given in paragraphs $2 a$ and $2 b$ in terms of an effect on the processing of the visual input, with two possibilities.

1. According to the hypothesis put forward in the introduction, the decrease in fixation duration might be due to the fact that in the space condition it was possible to know which region of the line had already been attended to and which had not, thus facilitating saccade programming. In the no-space condition, practically no feedback was provided about relative gaze position within the uni- form line of $x \mathrm{~s}$, so that an additional 30 - to 50-msec delay was needed at each fixation for elaboration of the " when" decision. What makes this hypothesis less attractive is the fact that it applies to changes only in fixation duration and cannot explain why saccade size would decrease in the condition in which saccade programming is supposed to be facilitated.

$2 a$. On the other hand, the observed effect on fixation durations could be explained in terms of lateral masking: In the space condition, lateral interferences between letters might have been reduced, so facilitating the visual processing. But if target letters were easier to find in the space condition, then this would predict a larger visual span here than in the no-space condition. Since assumption 1 predicts no changes in the size of the visual span as a function of the global spatial structure, assumption 2a can be tested against assumption 1 in an experiment that quantifies visual span for the different visibility conditions (see Experiment 2).

$2 \mathrm{~b}$. However, there is a second visual processing interpretation. Saccade size was smaller in the space condition than in the no-space condition. Hence, probably less information was extracted at each fixation in the space condition than in the no-space condition, and the processing of fewer letters per fixation might have sped up fixation durations. If this were true, and in agreement with the visual-span-control hypothesis and Jacobs's (1986) data, then one should find a smaller visual span in the space condition than in the no-space condition, contrary to what is expected under the lateral masking hypothesis given in 2a.

Finally, as can be seen from Table 1, search rate and total scanning time do change significantly as a function of target-background similarity. Because of the observed simultaneous decrease in fixation duration and saccade size, however, they do not change significantly in the space condition. This tradeoff in efficiency indicates that saccade programming is not facilitated in the space condition, a finding that weakens hypothesis 1 above.

\section{EXPERIMENT 2: VISUAL SPAN STUDY}

The results obtained in Experiment 1 would be understood better if the size of the visual span for the different conditions were known. As in O'Regan et al. (1983) and Jacobs (1986), visual span will be defined as the maximum eccentricity at which a given target letter can be detected within a given background with a certain probability. To measure visual span (Jacobs, 1986), Experiment 2 used a computer-controlled adaptive psychophysical procedure similar to the one known as "APE"' (Watt \& Andrews, 1981).

\section{Method}

Subjects. Five students and three members of our research lab participated in this experiment. All had normal vision and, except for one, were naive as to the purpose of the study. 
Stimuli, Apparatus, and Experimental design. Exactly the same stimuli were used here as in the preceding experiment (see Figure 1). Target letters were presented within a line of $x$ s on the computer screen. The line either was continuous (no-space condition) or was broken up into blocks of varying length (space condition), under the constraints outlined in Experiment 1. There was one single type of target letter (either of the two possible targets $c$ and $k$ ) used for a block of trials, and the subject was told what the target was. Thus, any effects due to confusion between the two possible targets were avoided. The apparatus was the same as in Experiment 1. So was the two-factor repeated measures design. There were four visibility conditions: easy $c$-target or difficult $k$-target; continuous background (no-space) or blocked background with interspersed spaces (see Figure 1).

Procedure. As in Experiment 1, the subject sat in an adjustable chair with his or her head stabilized by a chin/foreheadrest. At the beginning of each block, the subject fixated a small gap between two fixation lines and pressed a button to begin the trial. The stimulus line was then flashed on for $75 \mathrm{msec}$ (unmasked), and the subject gave his or her response using one of three buttons (target left, target right, or no target). The target letter, chosen for the given block, was presented at varying eccentricities from the fixation point, and the subject's task was to report if the target appeared to the right or to the left of the fixation point or if there was no target within the line of $x$ s.

The target eccentricities were chosen by the computer following the adaptive algorithm for measuring spatial visibility thresholds (visual span; Jacobs, 1986). At the beginning of each experimental block, the computer presented the target once at each of the extreme eccentricities $(-34,+34,-1$, and +1 character positions from the central fixation point, which was at position 40). Note that the eccentricities +35 and -35 were ruled out because we wanted to ensure lateral masking to be the same across eccentricities. This required that target letters always be flanked by at least one $x$. Note that this rule was also adhered to in the space condition, as mentioned in Experiment 1. On the basis of the responses given by the subject for each of the four extreme presentation eccentricities, the computer calculated a first estimation of the spatial threshold (eccentricity) corresponding to a response probability of $66.7 \%$, given the chance level of $1 / 3$. It then presented the target at the eccentricity resulting from this computation. The program used the "probit" technique (Finney, 1947; Watt \& Andrews, 1981) for fitting the data to a theoretical psychophysical curve. After the subject's next response, the program again computed the most probable threshold eccentricity, given all the data points sampled up to that moment, and fitted by the probit method. It then presented the target at the corresponding eccentricity, and so on. This procedure was repeated 50 times. The results in the literature, as well as simulations done by Humbert (1985), show that the precision of this adaptive method reaches a stable plateau by 50 trials. In total, 50 trials plus 25 randomly mixed-in control trials (without any target) and 75 training trials were used for one experimental block (for more details on the adaptive psychophysical procedure, see Humbert, 1985, or Jacobs, 1986).

\section{Results and Discussion}

For the theoretical reasons discussed by O'Regan et al. (1983) (see also Morrison \& Rayner, 1981), and for purposes of comparison with the data of Experiment 1, the values of visual span given in Table 2 are measured in number of letters identifiable on each side of the fixation point and not in degrees. Note that in the case of the space condition, spaces are counted as letters, as was done for the computation of saccade sizes in Experiment 1. Note also that, as in Jacobs (1986) and O'Regan et al. (1983), the "span" really is a half-span, since it refers to the ec-
Table 2

Visual Span (in Character Spaces) as a Function of Target-Background Similarity and Global Spatial Structure (Spaces) (8 Subjects)

\begin{tabular}{lcccc}
\hline & \multicolumn{4}{c}{ Experimental Condition } \\
\cline { 2 - 5 } & $\begin{array}{c}\text { (1) } \\
\text { Target } c \\
\text { No Spaces }\end{array}$ & $\begin{array}{c}\text { (2) } \\
\text { Target } c \\
\text { With Spaces }\end{array}$ & $\begin{array}{c}\text { Target } k \\
\text { No Spaces }\end{array}$ & $\begin{array}{c}\text { (4) } \\
\text { Target } k \\
\text { With Spaces }\end{array}$ \\
\hline Mean & 23.6 & 12.3 & 8.0 & 5.1 \\
$S E$ & 2.6 & 1.3 & .9 & .6 \\
\hline \multirow{5}{*}{$\begin{array}{l}\text { F(1,7): Factor target }=77.7, p<.0005 ; \\
\text { factor spaces }=36.4, p<.001 ; \\
\text { interaction }=9.9, p<.025 .\end{array}$} \\
\hline
\end{tabular}

Note-Visual span is defined as the maximum eccentricity (measured in number of character spaces from the fixation point), at which the target letter was reported correctly with a probability of $50 \%$ above chance level. The estimations of visual span were obtained by an adaptive psychophysical procedure, functioning in real time (see text).

centricity on either the left or the right side of the fixation point at which a target letter can be detected with $50 \%$ correct responses above chance level. The values of visual span given in Table 2 are obtained directly by the adaptive psychophysical method described above and correspond to the last threshold estimation given by the adaptive algorithm, which is the threshold corresponding to the 50th trial (control trials not counted).

Table 2 gives visual span as a function of graphic targetbackground similarity (target letter) and spatial structure (space and no-space). Measured in number of character spaces, span decreases systematically as targetbackground similarity increases $[F(1,7)=77.75$, $p<.0005]$. This result was expected and confirms the data obtained by Jacobs (1986). What is somewhat surprising is the second result, which shows that visual span strongly declines in the space condition when blank spaces are interspersed in the background of $x \mathrm{~s}[F(1,7)=36.4$, $p<.001]$. This result is consistent for both target letters. It indicates that the targets are easier to detect within a homogeneous spatial strucnure (no-space condition) than in a spatial structure that has several discontinuities because of the presence of the blanks. The blank spaces do not seem to significantly reduce lateral masking on an early (retinal or geniculate) level of processing, resulting in a facilitation of performance and thus in a larger visual span, as hypothesis $2 \mathrm{a}$ reasoned. Rather than reducing the lateral masking of the target caused by the $x \mathrm{~s}$, the presence of the blank spaces may inhibit higher order processes involved in target selection (see, e.g., the term "cognitive masking" in Eriksen \& Schultz, 1978). It is possible that the blank spaces act to divide visual attention (see Kahnemann \& Henik, 1977; Wolford \& Chambers, 1983; see also Mewhort, Marchetti, \& Campbell, 1982) and that, given the presentation time constraint and the limited capacity of the visual processing system, this degrades detection performance. Indeed, to do the task in the no-space condition, subjects need only to attend to something that is different from the homogeneous background. In the space condition, however, the presence of the blank spaces makes the task harder. 


\section{GENERAL DISCUSSION}

With regard to the different hypotheses put forward above to explain the scanning behavior data, the second result of the visual span experiment is crucial: A clearly smaller visual span is found in the space condition than in the no-space condition, for both targets. So, first, the data are against the "facilitation of saccade programming hypothesis" (see point 1 above), which predicted no change in visual span as a function of global spatial structure. Together with the data on search rate, this suggests that the use of a space structure does not facilitate search behavior because of a better orientation of the eye in the lines of $x \mathrm{~s}$. Second, the lateral masking hypothesis (see $2 \mathrm{a}$ above) can also be ruled out, since although it predicted a larger visual span for the space condition, the opposite result was observed. As pointed out above, the blank spaces seem to act as a sort of "cognitive mask" for target-selection processes rather than to reduce low-level masking mechanisms (in this kind of task).

So, as they concern the interpretation of the shorter fixation durations found in the space condition, the visual span results are clearly in favor of hypothesis $2 b$ above: Since both visual span and mean saccade size are smaller in the space condition, less information is extracted there than in the no-space condition. Hence, the result in the space condition is less processing time and shorter fixation durations. Concerning the data on saccade size, the visual span results indicate that there is no need to postulate a mixed scanning mode that combines "visual span control" and "block control." In fact, a pure visual-spancontrol strategy can account for the eye-movement data in this task. As can be seen from a comparison between mean saccade sizes (Table 1) and the values of visual span (Table 2) for the different visibility conditions, the data are in very good agreement (the coefficient of determination $r^{2}$ here is 0.99 ). Even when absolute values are compared, the data look quite similar, except that for Condition 1 ( $c$, no space), mean saccade size is about five letters smaller than the estimated visual span.

In sum, the use of a global spatial stimulus structure that is similar to the one we are familiar with in a reading situation does not facilitate scanning behavior in such a search task. The reason for this seems to lie in the processing of the visual input rather than in processes related to saccade programming: if blank spaces are interspersed within a homogeneous background of $x \mathrm{~s}$, thus creating character blocks of varying length, visual span decreases relative to that found with a completely uniform background. This causes a decrease in saccade size and, since less information is extracted during a single fixation, also a decrease in fixation duration.

However, as to the latter point, it should be noted that a simple quantitative model that equates mean fixation duration as a direct function of the size of the visual span (or of the mean number of letters skipped by saccades) cannot hold; otherwise one would find much longer fixation durations for the $c$ target than for the $k$ target, but the opposite is true. The ease of processing must be taken into account as a second important factor that acts on fixation duration. A tentative process model that can account for the observed effects on both eye-movement parameters has been presented recently (Jacobs, 1987). In accordance with current theorizing (Becker \& Jürgens, 1979; Findlay, 1983; Morrison, 1984; Rayner \& Pollatsek, 1981), the working model assumes two hypothetical subsystems that are involved in saccade control and function in parallel: the "when" system, which is supposed to determine the moment of occurrence of a saccade, and the "where"' system, which is supposed to calculate the saccade amplitude. The basic idea underlying the working model is that within the "when" system a decisional process is activated by the visual input that determines whether the sought-for target is present or not within the fixated area of the line (that is, within the limits of the visual span). It is assumed that in this kind of task the cognitive event triggering a saccade, which is one of the major mysteries in the study of eye-movement control (McConkie, Zola, \& Blanchard, 1984), is in the achievement of this decisional process. The preset decision thresholds increase as a function of target-background similarity, and so does mean fixation duration. While this decisional process is going on in the "when" system, the "where" system computes the amplitude of the next saccade on the basis of an internal representation of the visual span of the sought-for target. The model thus includes a non-sensory amplitude-computation channel within the "where" system, without which the present data cannot be explained. The accuracy or consistency (from one fixation to the next) of the adjustment of saccade amplitude to the anticipated visual span of the target is largely determined by the moment the "when" system triggers the saccade; once the "when" decision is elaborated, a saccade is irrevocably emitted, according to this model, whether the amplitude computation is terminated or not. The greater variability of saccade sizes in conditions in which visual span is relatively large and fixation duration short can accordingly be explained (see Jacobs, 1987, for more details).

A final consideration concerning the generalizability of the present data as well as future developments is the following: The various studies mentioned in the introduction have shown that, contrary to the present task, in reading, the interword blank spaces facilitate reading rate, probably because of their role in the perceptual isolation of word units and in saccade preparation. We know that, in reading, saccade control is not determined directly by visual span, but by higher order cognitive factors acting within these spatial sensory constraints (O'Regan et al., 1983). It therefore seems plausible that only in situations in which saccade size is directly computed on the basis of the visual span that corresponds to the stimulus condition, as in the present study, the presence of blank spaces does not facilitate eye-movement control. One way to test this would be to redo the present experiments using different levels of homogeneity of background to see where 
blank spaces facilitated search and where they did not. In light of the present results, the experiment by LévySchoen, O'Regan, Jacobs, and Coëffé (1984), which used random letters as a background, but without blank spaces, suggests indeed that, with such a highly heterogeneous pattern, blank spaces might have a facilitatory effect on search rate. In agreement with the above assumption, in the latter study, saccade size did not depend directly on visual span but was reduced to about five letters independently of the visibility condition, probably due to the limited number of different letters that can be processed within a single fixation. If blank spaces broke up the line pattern into perceptual units of about five letters, on average, as in the present study, this might facilitate eyemovement control in such conditions. A second possible development of the present experiments concerns the question of whether fixation duration can be reduced when visual cues are added to the repetitive background pattern, allowing feedback about relative gaze position within the line, thus facilitating saccade programming. The problem here will be to find cues that neither attract attention away from the target positions nor divide attention and reduce visual span in a way that the blank spaces probably did in this study.

\section{REFERENCES}

BECKER, W., JürGENs, R. (1979). An analysis of the saccadic system by means of double step stimuli. Vision Research, 19, 967-983.

BRADY, M. (1981). Toward a computational theory of early visual processing in reading. Visible Language, 15, 183-215.

ERIKSEN, C. W., \& SCHULz, D. W. (1978). Temporal factors in visual information processing: A tutorial review. In J. Requin (Ed.), Attention and performance, VII. Hillsdale, NJ: Erlbaum.

FindLAY, J. M. (1983). Visual information processing for saccadic eye movements. In A. Hein \& M. Jeannerod (Eds.), Spatially oriented behavior. New York: Springer.

Finney, D. J. (1947). Probit analysis. Cambridge: University Press.

FISHER, D. F. (1975). Reading and visual search. Memory \& Cognition, 3, 188-196.

Humbert, R. (1985). Comparaison par simulation de méthodes adaptatives de mesure de seuil. Bulletin de la Société Française d'Optique Physiologique, 4, 2.

JaCoBs, A. M. (1986). Eye movement control in visual search: How direct is visual span control? Perception \& Psychophysics, 39, 47-58.

JACOBS, A. M. (1987). Towards a model of eye movement control in visual search. In J. K. O'Regan \& A. Lévy-Schoen (Eds.), Eye movements: From physiology to cognition (pp. 275-284). Amsterdam: North-Holland.

JaCobs, A. M., \& O'RegaN, J. K. (in press). Spatial and/or temporal adjustments of scanning behavior to visibility changes. Acta Psychologica.

Kahnemann, D., HENIK, A. (1977). Effects of visual grouping on immediate recall and selective attention. In S. Dornic (Ed.), Attention and performance, VI (pp. 307-332). Hillsdale, NJ: Erlbaum.

Lévy-Schoen, A., O'Regan, J. K., JaCobs, A. M., \& Cö̈FFé, C. (1984). The relation between visibility span and eye movements in various scanning tasks. In A. G. Gale \& F. Johnson (Eds.), Theoretical and applied aspects of eye movement research. Amsterdam: North-Holland.
MCCoNKIE, G. W. (1983). Eye movements and perception during reading. In K. Rayner (Ed.), Eye movements in reading: Perceptual and language processes (pp. 3-30). New York: Academic Press.

MCCONXIE, G. W., \& ZoLA, D. (1984). Eye movement control during reading: The effect of word units. In W. Prinz \& A. F. Sanders (Eds.), Cognition and motor processes. Berlin: Springer.

McConkie, G. W., Zola, D., \& Blanchard, H. (1984). What is the basis for making an eye movement during reading? In A. G. Gale \& F. Johnson (Eds.), Theoretical and applied aspects of eye movement research (pp. 169-178). Amsterdam: North-Holland.

MENZ, C., \& Groner, R. (1985). Experiments on visual orientation in reading: Effects of lining, letter spacing and inserted elements. In R. Groner, G. W. McConkie, \& C. Menz (Eds.), Eye movements and human information processing (pp. 183-196). Amsterdam: NorthHolland.

Mewhort, D. J. K., Marchetti, F. M., CAmpbell, A. J. (1982). Blank characters in tachistoscopic recognition: Space has both a symbolic and a sensory role. Canadian Joumal of Psychology, 36, 559-575.

MofrTt, K. (1980). Evaluation of the fixation duration in visual search. Perception \& Psychophysics, 27, 360-372.

Morrison, R. E. (1984). Manipulations of stimulus onset delay in reading: Evidence for parallel programming of saccades. Journal of $E x-$ perimental Psychology: Human Perception \& Performance, 10, 667-682.

Morrison, R. E., \& RAYNER, K. (1981). Saccade size in reading depends upon character spaces and not visual angle. Perception \& Psychophysics, 30, 395-396.

NATtKemPER, D., \& Prinz, W. (1987). Determinants of fixation duration in continuous search. In J. K. O'Regan \& A. Lévy-Schoen (Eds.), Eye movements: From physiology to cognition. Amsterdam: NorthHolland.

O'Regan, J. K. (1979). Saccade size control in reading: Evidence for the linguistic control hypothesis. Perception \& Psychophysics, 25, 501-509.

O'RegAN, J. K., \& Lévy-SChOEN, A. (Eds.) (1987). Eye movements: From physiology to cognition. Amsterdam: North-Holland.

O'Regan, J. K., Lévy-Schoen, A., \& JacoBs, A. M. (1983). The effect of visibility on eye movement parameters in reading. Perception \& Psychophysics, 34, 457-464.

Pollatsek, A., \& RAYNer, K. (1982). Eye movement control in reading: The role of word boundaries. Journal of Experimental Psychology: Human Perception \& Performance, 8, 817-833.

RAYNER, K. (1984). Visual selection in reading, picture perception and visual search. In H. Bouma \& D. G. Bouwhuis (Eds.), Attention and performance $X$ (pp. 67-96). Hillsdale, NI: Erlbaum.

RAYNer, K., \& Fisher, D. L. (1987). Eye movements and the perceptual span during visual search. In J. K. O'Regan \& A. Lévy-Schoen (Eds.), Eye movements: From physiology to cognition. Amsterdam: North-Holland.

Rayner, K., Pollatsek, A. (1981). Eye movement control during reading: Evidence for direct control. Quarterly Journal of Experimental Psychology, 33A, 351-373.

WatT, R. J., \& ANDrews, D. P. (1981). APE: Adaptive Probit Estimation of psychometric function. Current Psychological Reviews, 1 , 205-214.

Wolford, G., CHAMBers, L. (1983). Lateral masking as a function of spacing. Perception \& Psychophysics, 33, 129-138.

(Manuscript received July 7, 1986; revision accepted for publication January 16, 1987.) 